\title{
Design and Analysis of a New Space Telescopic Mast
}

\author{
Zhao Yang, Yong Xiao, Lipeng Wang \\ China Academy of Space Technology, 710000 Xi'an, China
}

\begin{abstract}
The space telescopic mast has the characteristics of high rigidity, strong bearing capacity and simple deployment principle. Aiming at the shortcomings of large mass and limited screw length in the existing telescopic mast, a spiral climbing-driven telescopic mast was designed, and the structural design and kinematic analysis were studied. The verification results of the motion simulation through ADAMS software are consistent with theoretical calculations. The new telescopic mast has the characteristics of light weight, low manufacturing cost, and unrestricted length of single-section stretching.
\end{abstract}

\section{Introduction}

The space extension mechanism is an important part of the spacecraft and mainly has two functions:one is to support the antenna structure; the other is to increase the distance between the antenna and the satellite body to reduce their interference. The stretching mechanism facilitates transportation and storage, saves mass and volume, and has the advantage of carrying greater loads in the launching state. The telescopic mast is a common space stretching mechanism, composed of a series of coaxial thin-walled tubes nested in each other. It can be deployed by a drive device (such as a screw nut, a rope, etc.) to finally achieve the stretching function ${ }^{[1]}$.

The telescopic mast has a simple principle, a large carrying capacity and a high reliability, and is suitable for the space stretching of medium and short distances. The telescopic mast is classified into a screw driven type, a rope driven type and a helical drum type according to different driving modes. The screw type is the most widely used telescopic mast. Zhong Bowen [2] designed and analyzed the screw-type telescopic mast. The length of the single-screw drive mode is limited by the length of the screw, and the screw has a large mass and high cost. The American Northrop Grumman Company and Germany's Dormier Company are in the leading position in the industry. Lin Changmin ${ }^{[3]}$ proposed a screw-type and rope-driven telescopic mast. The inherent safety and reliability of the rope-driven system is poor, and the driving power is small. Li Changzhou ${ }^{[4]}$ proposed a screwtype driving mechanism. The screw-cylinder drive mode has drive cylinders, large gears, and other structural components, which are of higher quality and require more payloads.

In this paper, a telescopic mast with a new type of driving mechanism is designed, and the stretching of the system is achieved by stepwise extension of the mast.

\section{Structural design of the mechanism}

\subsection{Design ideas}

As shown in Fig 1(a), the rack and pinion mechanism rotates the gear along the rack. When the trajectory line of the rack is a helix, the gear can still move spirally along the rack, as shown in Fig. 1(b). For the purpose of improving system stability, the drive gear is designed as a dual-gear spiral rack mechanism, similar to the planetary gear structure, as shown in Fig. 1(c). Due to the complex design of the tooth profile of the helical rack, it is extremely difficult to manufacture and the cost is high. Therefore, the rack and pinion mechanism is designed

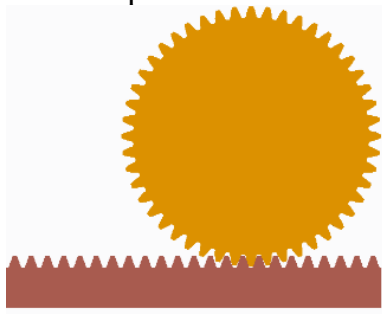

(a) The schematic diagram of rack and gear

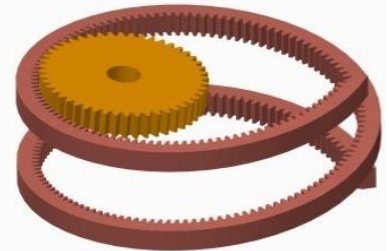

(b)The schematic diagram of spiral rack and gear 


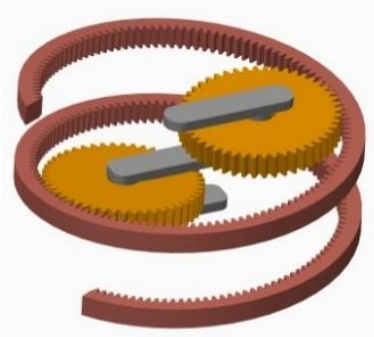

(c) The schematic diagram of spiral rack and double gears

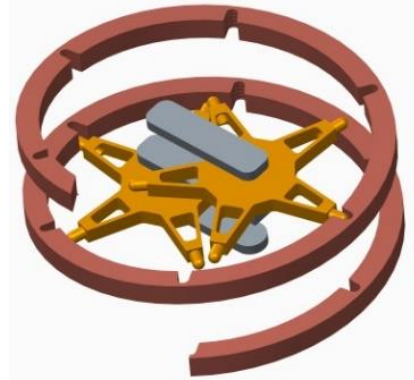

(d) The schematic diagram discrete gears and rack

Figure 1. The design ideas of driving mechanism

as the discrete transmission of driving pin and transmission hole, as shown in Fig. 1(d). The generatrix of the transmission hole is simulated according to MATLAB.

\subsection{New structural system design}

The new telescopic mast is mainly composed of two modules: the stretching mechanism and the driving mechanism. The stretching mechanism includes a stretching rib, a middle rib, and a fixed tube. The driving mechanism includes a crank, a climbing wheel, a driving pin, a locking mechanism and other components, and is a core part of the new mechanism. Fig. 2 is a schematic structural diagram of a new type of telescopic mast.
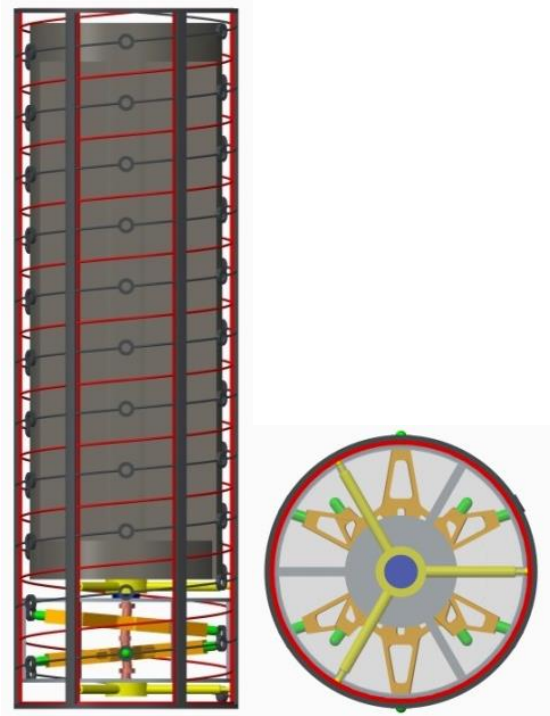

Figure 2. The structure diagram of stretching mechanism

\subsection{Driving mechanism design}

The driving mechanism includes a bearing, a crank, a climbing wheel, a support base, a driving pin, and a locking mechanism, just as shown in Fig. 3. The motor rotates the crank which is connected with the climbing wheel through the bearing. The driving pin on the rim of the climbing wheel comes into contact with the transmission hole on the inner wall of the fixed tube, and an enveloping transmission occurs to drive the climbing wheel to spiral up, thereby driving the mast to extend. The upper and lower locking mechanism is mainly responsible for the axial positioning and locking of the mast.

\subsection{Stretching mechanism design}

The stretching mechanism is designed as a three-stage mechanism, which is composed of a stretching rib, a middle rib, and a fixed tube. The outermost mast always remains fixed, called the fixed tube. During the stretching process, the driving pin always meshes with the driving hole on the fixed tube. The middle rib is hollow frame structures. The driving mechanism is always in contact with the driving pin of the fixed tube to avoid interference with the extension ribs and plays a role in weight reduction. Guide grooves are provided inside the fixed and middle rib to position the movement direction. There

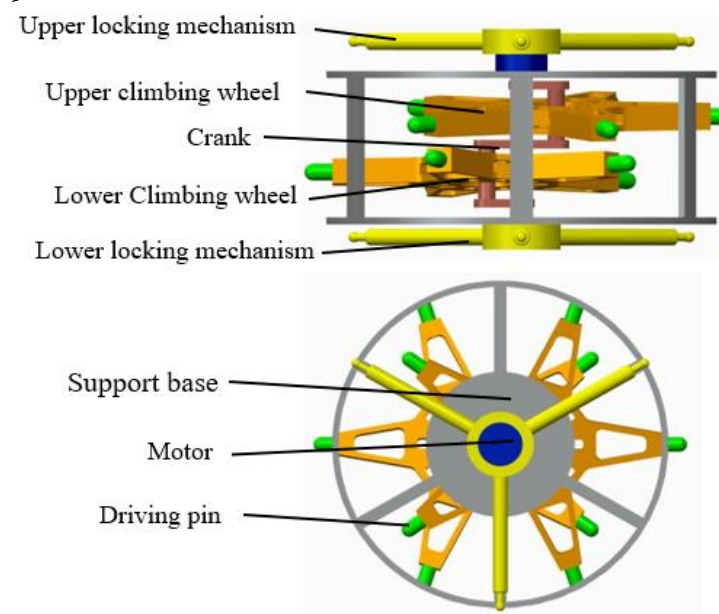

Figure 3. The driving mechanism

are three locking taper holes at the top and bottom of the middle rib. The upper locking mechanism and the upper taper hole of the middle tube are locked. Three locking cone holes are uniformly arranged on the top of the fixed tube, and the lower locking mechanism is locked.

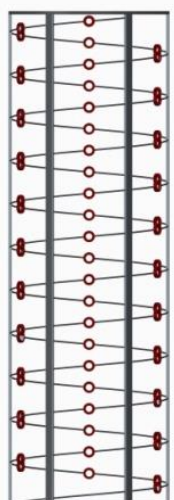

(a)Fixed tube

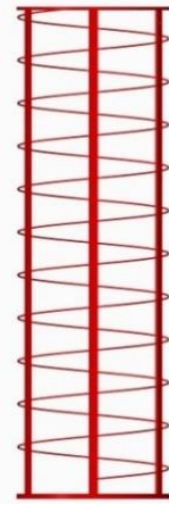

(b) Middle rib

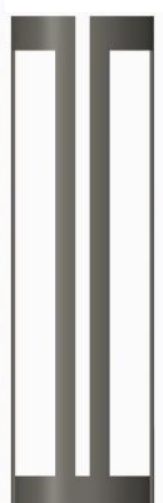

(c) Stretching rib
Figure 4. Mast structure diagram 


\subsection{Stretching process analysis}

During the working process of the telescopic mast, the driving mechanism is driven by the motor. The masts at all levels are locked by the locking mechanism, and are nested in sequence. The unfolding process is divided into following four steps. The schematic diagram is shown in Fig .5.

The first step: As shown in Fig. 5(a), the system is in a contracted state in its initial state. When it starts to stretch, the drive mechanism rises. When the stretching rib is pushed to the top of the middle rib, the motor is stopped by the stroke switch .The stretching rib and the middle rib are locked by the upper locking mechanism, as shown in Fig. 5(b).

The second step: the motor reverses while the driving mechanism moves downwards. The descending process is the reverse process of climbing. At this time, the stretching rib is locked by the middle rib through the upper locking mechanism, and the driving mechanism is separated from the stretching rib. When it reaches the root, the motor stops moving and the driving mechanism is locked through the lower locking mechanism. As shown in Fig. 5(c).

The third step: the motor rotates forward, the driving mechanism climbs, and the middle rib rises. When climbing to the top, it stops moving. The middle rib and the fixed tube are locked by the lower locking mechanism, and the three-level stretching is completed. As shown in Fig. 5(d).

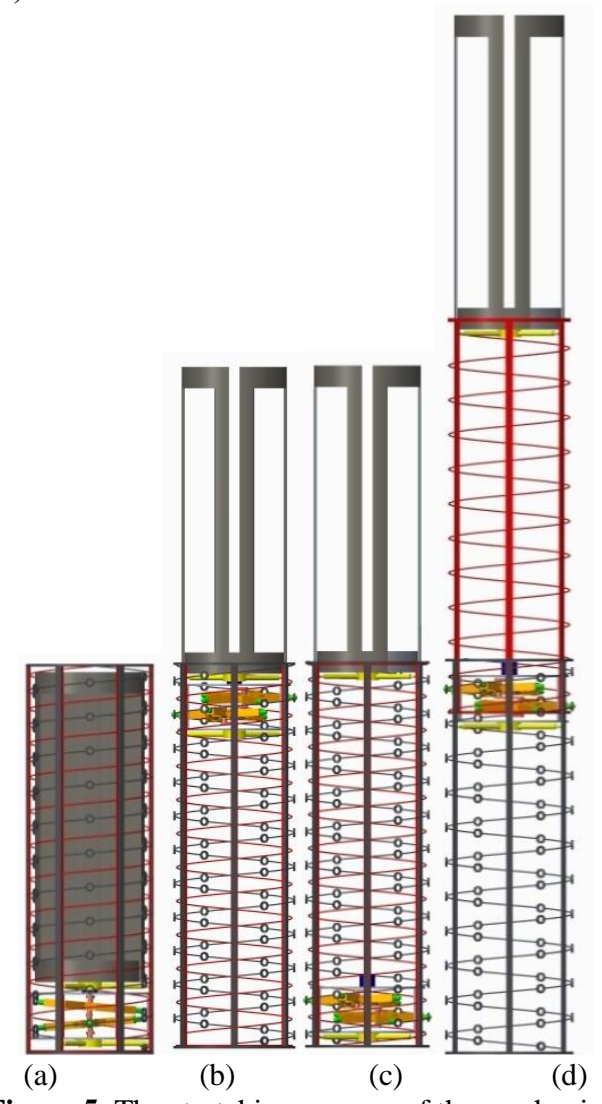

Figure 5. The stretching process of the mechanism

During the moving process, the driving mechanism is always in contact with the fixed tube. The middle rib only needs to leave enough space.

\section{Kinematic analysis}

The movement of the stretching mechanism system is relatively complex, involving a plurality of movement states such as translation and rotation. This section analyzes the motion process of the system, establishes the system motion equations through kinematics related theory, and draws its motion track diagram through MATLAB.

\subsection{Plane motion analysis}

The two-dimensional planar kinematics of the drive system is analyzed, as shown in Fig. 6. The system is simplified as a crank linkage, taking coordinate system $S_{0}\left(x_{0}, y_{0}\right)$, relative coordinate system $S_{1}\left(x_{1}, y_{1}\right)$.

The angular velocity of the driving pin relative to the crank is ${ }^{\omega_{1}}$, the average angular velocity relative to the rotational center of the fixed tube is ${ }^{\omega_{2}}$, and the angular velocity of the crank relative to the support base is ${ }^{\omega_{3}}$.In order to ensure a constant transmission ratio, the system can be driven smoothly. Here, the transmission ratio ${ }^{n}$ is:

$$
n=\frac{\omega_{2}}{\omega_{3}}
$$

When an enveloping transmission occurs between the driving pin and the transmission hole, the crank hole, the crank connecting rod moves from position 1 to position 5 . The crank rotates through the angle $2 \theta$ so that the rotated angle range is $[-\theta, \theta]$, and the connecting rod rotates through the angle ${ }^{[-\theta / n, \theta / n]}$.

When the crank is in position 1, the included angle is $\theta$, at which point the driving pin enters the transmission hole and engages with the surface of the envelope surface A. When $\theta=0$, the crank is in line with the connecting rod. When the corner is ${ }^{[-\theta, 0]}$, the crank is engaged with the surface $\mathrm{B}$. When the rotation angle is $-\theta$, the driving pin disengages from the transmission envelope hole. As shown in Fig. 6, the crank links sequentially turn to the illustrated positions $2,3,4,5$.

Set the relative coordinate of any point on the connecting rod to $\left[\mathrm{X}_{1}, \mathrm{Y}_{1}\right]$, and according to the coordinate transformation formula, the absolute coordinate of the point on the connecting rod is formula (2):

$$
\left[\begin{array}{l}
x_{0} \\
y_{0}
\end{array}\right]=\left[\begin{array}{cc}
\cos \frac{\theta}{n} & \sin \frac{\theta}{n} \\
-\sin \frac{\theta}{n} & \cos \frac{\theta}{n}
\end{array}\right]\left[\begin{array}{l}
x_{1} \\
y_{1}
\end{array}\right]+r\left[\begin{array}{c}
\cos \theta \\
\sin \theta
\end{array}\right]
$$




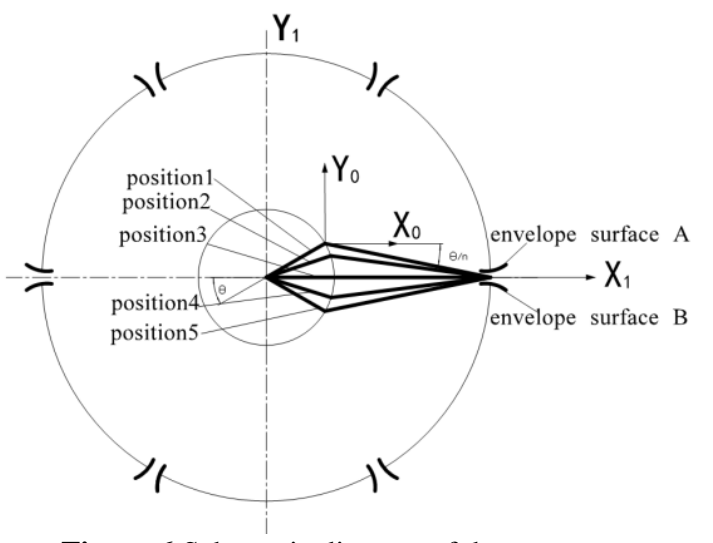

Figure.6 Schematic diagram of the movement

Set the geometric center of the driving pin to point A and the relative coordinate to ${ }^{[l, 0]}$, then substitute the absolute coordinate as:

$$
\left[\cos \frac{\theta}{n} l+r \cos \theta, \sin \frac{\theta}{n} l+r \sin \theta\right]
$$

When $n=5, r=40$, and $l=200$, the planar motion trajectory at this time is shown in Fig. 7(a). When the system has an inclination angle, the spatial movement trajectory is a spiral rising curve, and the sharp corner is in the position of the transmission meshing hole. The schematic diagram is shown in Fig. 7(b).

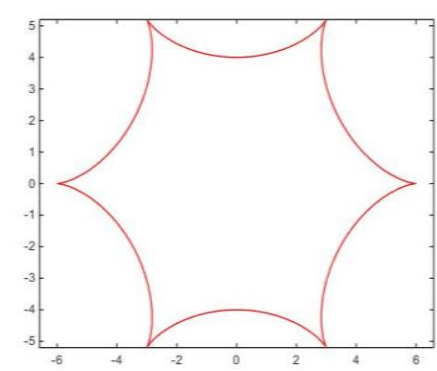

(a) Plane movement track diagram

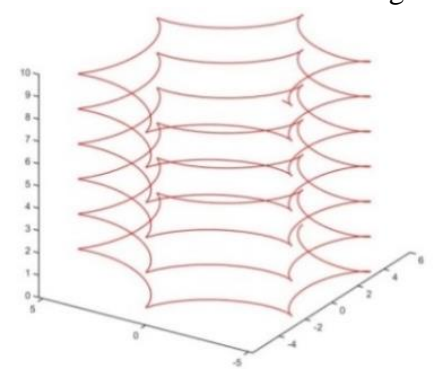

(b) Space movement track diagram Figure 7

\subsection{Spatial motion analysis}

The trajectory of the space is similar to that of a cylindrical helix, and its helical inclination is equal to that of the crank. The diameter of the geometric center of the driving pin is $\mathrm{D}$, the angle of inclination of the crank, and the distance between adjacent holes in the vertical position is set as the lead of the space-like helix:

$$
H=\tan \theta \cdot D \cdot \pi
$$

$\mathrm{N}+1$ holes are uniformly distributed in each lead. The difference in height between adjacent holes is:

$$
\Delta z=\frac{1}{n+1} H
$$

According to kinematics, the transmission ratio is:

$$
n=\frac{\omega_{3}}{\omega_{1}-\omega_{3}}
$$

The time that the driving pin completes an enveloping motion is $t$, and then $t$ is

$$
t=\frac{\pi}{n \omega_{1}}
$$

Drive mechanism's climb speed:

$$
V_{h}=\frac{\Delta z}{t}
$$

\subsection{Envelope numerical simulation}

In the meshing transmission between the driving pin and the transmission hole, the trajectory of the pin hole is determined by the shape of the transmission hole. According to the relevant literature, the drive pin can be selected to be spherical. ${ }^{[5]}$ In order to ensure that the climbing wheel moves according to the established trajectory, it is necessary to solve the pinhole outer envelope bus ${ }^{[6]}$ MATLAB is selected to solve the envelope numerically. The simulation idea is to simulate the movement trajectory of the driving pin at different moments and the maximum outsourcing envelope of the movement trajectory is the bus of the transmission pin. Fig. 8 shows the MATLAB numerical simulation curve.

\section{Kinematics simulation}

Stretching mechanism structure and form of movement is complex. After the structural design and motion analysis were completed, the feasibility of the

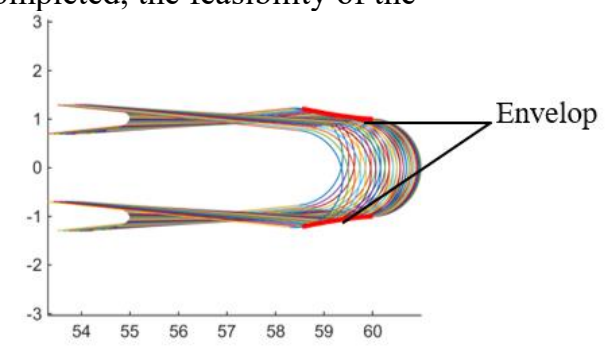

Figure 8. Envelope numerical simulation

system was analyzed and verified using ADAMS software and the feasibility of the model was verified Creo2.0 modeling and assembly, data exchange with ADAMS through Parasolid format, after the introduction of constraints to add ${ }^{[7]}$. According to the motion relationship, the constraints that the system mainly needs to add are:

Fixed pair (joint1): fixed tube relative to the ground;

Mobile pair (joint2): supporting seat relative fixed tube; Rotary pair (joint3): crank relative support seat;

Rotary pair (joint4): climbing wheel 1 Relative crank; Rotary pair (joint5); climbing wheel 2 Relative crank Set the system speed based on the spatial kinematics related equations. Assuming that the $\omega_{1}$ is $1.2 \% \mathrm{~s}, \omega_{3}$ is 
$1 \%$, and the moving speed $V_{h}$ is $0.7387 \mathrm{~mm} / \mathrm{s}$. Kinematic simulations are performed according to the established simulation speed. The calculated trajectories are consistent, verifying the correctness and feasibility of the design. The simulation diagram is shown in Fig. 9.

\section{Conclusion}

(1) The telescopic mast with different driving modes is briefly analyzed, and its characteristics are summarized and analyzed;

(2) A new type of telescopic mast was designed to perform structural modeling and analysis of the new mechanism. The envelope is solved through the kinematics analysis and the use of numerical simulation method ;

(3) Using ADAMS software for kinematics simulation, motion simulation and theoretical calculations are consistent, verifying the correctness and feasibility of the design.
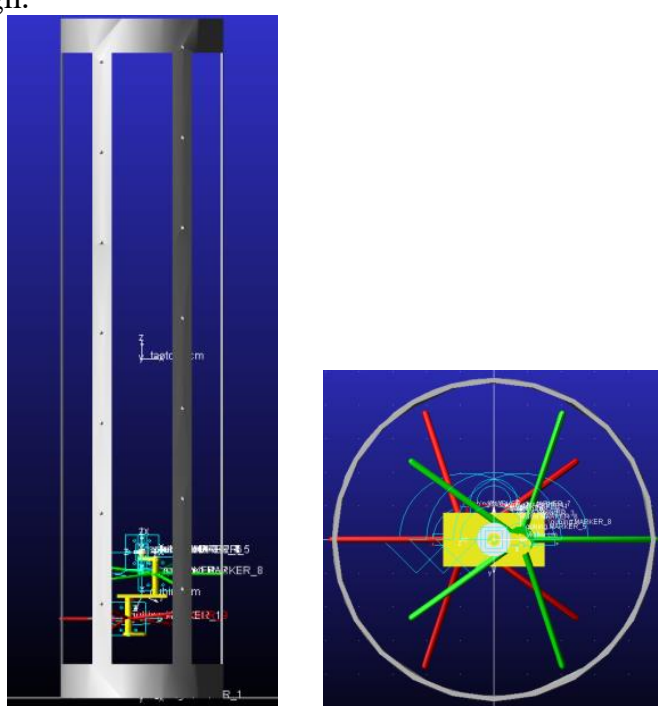

Figure 9. ADAMS kinematics simulation model

\section{References}

1. Zhiquan Liu,Biao Li,Gang Cheng. Review of Deployable-truss Masts for Space Application[J] . Chinese Space Science and Technology,2011,4(2) :32-38.

2. Bowen Zhong. The Design and Analysis of Telescopic Mast[D]. Harbin Institute of Technology,2006.

3. Shangmin Lin. Structural Design and Analysis of Space Deployable Mast[D]. Xi'an University of Electronic Science and Technology,2012.

4. Changzhou Li Design and Analysis of Space deployable Cable-drive Telescopic Mechanism[D].Harbin Institute of Technology,2016.

5. Ruiliang Chen. The Optimization of Inner Meshed Gear Pump with Wedge and External Circle-arc and its Envelope Teeth[D].Fuzhou University. 2003.
6. Dekun Feng,Xiangfeng Ma. Enveloping Principle and its Application in Machinery[M]. Machinery Industry Press, 1994.

7. WuyunZhao, Zenglu Shi,Fei Dai, Xuejun Zhang. ADAMS 2013 Fundamentals and Application Examples Tutorial[M]. Tsinghua University Press, 2015. 